\title{
ENTRE MÓVILES Y REDES. LAS PROPUESTAS EDUCATIVAS MEDIADAS POR DISPOSITIVOS DIGITALES MÓVILES Y REDES SEMÁNTICAS.
}

\author{
Silvia Coicaud (Universidad Nacional de la Patagonia San Juan Bosco)* \\ scoicaud@unpata.edu.ar
}

Recibido: 24/06/2011 Aceptado: 26/10/2011

\begin{abstract}
Resumen
Asistimos a complejos cambios en todos los niveles del sistema educativo a partir de la incorporación de las tecnologías de la información y la comunicación. Actualmente, la tendencia consiste en la fusión de servicios y funciones, y los dispositivos móviles adquieren relevancia en relación a su masividad de uso en las culturas juveniles. Esto instaura nuevos desafíos para la enseñanza, pero al mismo tiempo genera la necesidad de reflexionar críticamente acerca del rol de la escuela respecto y los procesos de enculturación de los niños y jóvenes. La evolución en los alcances de la red Internet, y sus enormes posibilidades para la educación, es otro aspecto central. No obstante, resulta imprescindible analizar estos cambios en el contexto complejo en el que tienen lugar los procesos de enseñanza y aprendizaje, dejando de lado posturas míticas y reduccionistas. Esto será posible en el marco de propuestas sólidas de formación docente, en las cuales se aborde de manera integral una alfabetización múltiple, que profundice el metalenguaje de las tecnologías digitales considerando sus diversas manifestaciones.
\end{abstract}

\section{Palabras Clave}

Tecnologías - Sincretismo - M-learning - Alfabetización Múltiple - Internet.

\section{Abstract}

We assist with complex changes in all levels of education from the incorporation of information technologies and communication. Currently, the trend is the merging of services and functions, and mobile devices become relevant in relation to its massive

* Doctora en Didáctica y Organización Escolar. Universidad de Granada. Magister en Educación Superior. Universidad Nacional de la Patagonia San Juan Bosco. 
use in youth cultures. This introduces new challenges for teaching, but also generates the need to think critically about the role of the school and processes of enculturation of children and youth. The evolution in the scope of Internet and its enormous potential for education is another key aspect. However, it is essential to analyze these changes in the complex context in which processes of teaching and learning occur, resigning to mythical and reductionist positions. This will be possible in the context of solid proposals of teacher training, with comprehensively approach multiple literacy, to deepen the metalanguage of digital technologies considering its various manifestations.

\section{Key words}

Technologies - Syncretism - M-learning - Multiple Literacy - Internet.

\section{El desafío de incrementar mentes y realidades a través de nuevos dispositivos tecnológicos}

Las perspectivas que las tecnologías de la información y la comunicación nos presentan para las próximas décadas, implican un avance importante hacia los dispositivos móviles. De este modo, el e-learning y el b-learning trasvasarán sus alcances y expectativas para convertirse cada vez más en m-learning. Las tecnologías móviles atribuirán un carácter nómade a las escuelas (Coll y Monereo, 2008) y las mismas posibilitarán promover en mayor medida la comunicación fluida entre educadores y estudiantes de distintos lugares del planeta. Las funciones de las tecnologías móviles de comunicación que se desarrollaron de manera autónoma -telefonía, multimedia, localización espacial, computadoras de bolsillo, etc-, se están fusionando para multiplicar sus potencialidades. Las "aulas de informática" instaladas en nuestras instituciones educativas obedecen a una concepción pedagógica y tecnológica retrógrada, pues se sustentan en los viejos cánones de poder, control y enclaustramiento que dieron origen a la mayor parte de las instituciones en la sociedad. Resulta fundamental que las TIC adquieran ubicuidad en las instituciones, garantizándose su acceso a toda la comunidad educativa en todo momento y lugar. Si bien aún no se ha generalizado su uso en los ámbitos educativos, las experiencias desarrolladas demuestran que los recientes dispositivos móviles que sincretizan funciones informáticas y comunicacionales diversas, permiten una utilización personalizada de herramientas tecnológicas por parte de los estudiantes, propiciando situaciones altamente motivadoras de aprendizaje cooperativo.

L. Fortunati (1997) observa que la dimensión ambigua de la presencia y/o ausencia en el espacio conlleva a reestructurar el sentido de pertenencia a un lugar. De este modo, cuando las personas interactúan con este tipo de tecnologías la construcción de este sentido de pertenencia se transforma, a partir de la conformación de una red de comunicaciones. Ejemplo de ello son las situaciones cotidianas en las cuales los jóvenes se consideran presentes en 
un encuentro social si permanecen en contacto entre ellos a través de mensajes de texto o del facebook.

Pero no podemos dejar de considerar que son numerosas las paradojas, los mitos y los prejuicios relacionados con las tecnologías en la sociedad. Entre los mitos y pre-concepciones más frecuentes que circulan, podemos mencionar la idea de neutralidad de las producciones científicas y tecnológicas; la ilusión de lograr el perfeccionamiento de la democracia mediante el desarrollo de nuevas tecnologías; la creencia en la autonomía de las máquinas respecto de la intervención humana; la confianza puesta en el progreso y la mejora constante a partir de las invenciones; la utopía de que habrá una transferencia universal de los avances y beneficios tecnológicos a todos los lugares del mundo, mejorando la calidad de vida de todas las personas; el pensamiento acerca del logro de una tecnología única y unívoca; la apuesta por el poder supremo de los artefactos, y el valor de la eficacia por encima de cualquier otro.

También somos conscientes de que existe polarización en muchas regiones del mundo entre quienes gozan de un acceso completo a todas las tecnologías disponibles, y quienes están fuera de esta posibilidad. Es decir, entre "infonautas" e "infoparias", neologismos que califican claramente las nuevas divisiones sociales que se vienen sumando a otras ya existentes. La ilusión posmoderna de una "generación digital" es un estereotipo cristalizado que solapa las verdaderas dificultades y frustraciones que tienen muchos niños y jóvenes por quedar afuera de la posibilidad del consumo de tecnologías. Esto origina procesos de violencia real y de violencia simbólica en muchas escuelas a las que asisten alumnos de clases sociales marginadas, por la insatisfacción de los jóvenes que no pueden acceder a un teléfono móvil o a una computadora, por ejemplo.

Ha sido muy difundida la clasificación dicotómica de M. Prensky (2001) respecto a la existencia de "nativos e inmigrantes digitales". Para los nativos digitales las personas inteligentes son aquellas que logran acceder con mayor facilidad a la información, a través de una red amplia de contactos y fuentes disponibles. Piensan que el conocimiento no está en la "cabeza", sino que se encuentra distribuido en múltiples sitios, dispositivos y artefactos (computadoras, teléfonos móviles, televisión, archivos diversos, links específicos, libros, etc) y consideran que quienes pueden disponer y dar cuenta del mismo de manera rápida y eficiente resolviendo problemas de manera creativa y compartiendo sus hallazgos, son gente competente. Juzgan a las personas por lo que expresan y producen en las redes digitales, despreocupándose por su pertenencia a determinada cultura, género o localización geográfica. La sincronicidad en la comunicación y la posibilidad de una rápida sociabilidad son los servicios de Internet que responden en mayor medida a sus intereses, por lo cual facebook en la actualidad -y el chat hace poco tiempo atrás- constituyen la herramienta de la red que más valoran y utilizan. Sin embargo, los nativos tienen dificultades para procesar información relevante. Sus habilidades de búsqueda y las estrategias de selección de fuentes válidas que utilizan suelen ser poco sofisticadas. Una 
respuesta típica de los adolescentes cuando se les pide un juicio acerca de la validez de un dato es: "está en Internet".

Para los inmigrantes digitales, en cambio, se postula que el conocimiento es concebido como "situado" en la mente individual, siendo la memoria la función cognitiva que posibilita su acopio. La idea de "posesión" del conocimiento por parte de las personas es predominante. Los inmigrantes utilizan lenguajes caracterizados por su linealidad secuencial, a través de una lógica de comprensión y producción que no aborda la globalidad y simultaneidad de los lenguajes audiovisuales, en los cuales se superponen de modo flexible e integrado símbolos gráficos, la voz, los textos, los sonidos, las animaciones, etc. A diferencia de los nativos, reconocen el valor de los conocimientos previos de los sujetos como criterio principal para la validación del conocimiento, y prefieren el trabajo asincrónico, ordenado y secuencializado con documentos, aún cuando utilicen soportes en red.

Si bien la tipología de Prensky está siendo ampliamente utilizada para caracterizar las particularidades que supuestamente adquieren las culturas juveniles actuales, la misma plantea una postura estereotipada y esquemática que deja fuera del análisis las complejas relaciones que tanto los jóvenes como los adultos construyen en los diferentes contextos sociales en los que les ha tocado vivir. Se da por descontado que los jóvenes menores de veinte años nacieron y crecieron usando el ratón de la computadora y que, por lo tanto, poseen habilidades tecnológicas digitales casi innatas. Este discurso ha sido el pivote sobre el cual las grandes corporaciones mediáticas han obtenido inconmensurables ganancias económicas. Empleada de manera dicotómica y reduccionista, la categoría de "nativos digitales" solapa las verdaderas necesidades y preocupaciones que tienen los jóvenes en la actualidad, considerándolos sólo como consumidores hedonistas desconectados de la realidad.

Dejando de lado enfoques desconocedores de las abismales diferencias sociales respecto al acceso a las tecnologías y las clasificaciones de los sujetos desde un determinismo sobresimplificador, debemos admitir sin embargo que en ambientes impregnados de tecnologías la mente humana está adquiriendo en forma progresiva otras significaciones. Ante la inquisidora demanda por saber si desde los comienzos de su irrupción en los ámbitos educativos las TIC han producido cambios sustanciales en los esquemas cognitivos de los sujetos, las investigaciones en el campo de la psicología cognitiva dan cuenta que seguramente existirán modificaciones microgenéticas relevantes en las nuevas generaciones de jóvenes (Salomon, 1992). La objetivación de los sistemas culturales constituye un proceso más pragmático que epistémico en estos sujetos, desde una pluralidad representacional que se construye a partir del uso integrado de diferentes códigos.

En relación con estas particularidades que distinguen la conformación de una mente virtual en los estudiantes de esta última década, resulta imprescin- 
dible analizar los modos de conformación de las tareas de aprendizaje. Las experiencias simuladas desde entornos digitales en campos específicos del conocimiento, por ejemplo, deberán ser planificadas con mayor asiduidad a partir de estos parámetros. Esto implica por parte de las instituciones educativas, optar por el reemplazo de prácticas artificiosas de "laboratorilandia" de un mundo escolar de apariencias en el que las verdaderas entidades son invisibles, improbables o escurridizas (Claxton, 1994) por instancias genuinas de aprendizaje que impliquen una aproximación significativa a lo real. Estos criterios contribuyen también a redefinir los modos de comunicación didáctica, pues los grupos de estudio modifican su constitución al integrarse virtualmente en ellos a través de Internet desde diversas regiones, e incluso desde diferentes culturas. La co-generación del conocimiento entre personas implicadas de múltiples maneras en los propósitos de la educación, constituye un proceso que cada vez adquiere mayor relevancia en nuestras instituciones. Estas "multitudes inteligentes" (Rheingold, 2004) surgidas desde las actuales redes tecnológicas conforman colectivos que trabajan de manera recíproca y colaborativa, en una suerte de puzzle organizado a partir de otras dinámicas de interacción humana que expanden su capacidad de percepción para abordar una nueva realidad incrementada.

Al respecto, resulta fundamental reconocer los aportes más sustanciales que realiza la psicología cognitiva relacionados con el desarrollo de las funciones superiores de la mente, en vinculación con las mismas. Desde este campo, se establece que es la propia actividad humana la que debe ser tomada como unidad de análisis, pues ella preserva en sí misma las propiedades de las totalidades complejas de la conciencia. Esta actividad no consiste sólo en respuestas o reflejos dados, sino que implica componentes de transformación del medio mediante la ayuda de diversos instrumentos: las herramientas y los signos. Los ámbitos culturales de los que formamos parte, son los que posibilitan esta interiorización de símbolos en nuestra conciencia. En la medida en que la cultura y la educación nos ofrezcan instrumentos valiosos para desarrollar nuestras actividades humanas, podremos crecer como personas y transformar la sociedad.

Estas transformaciones están teniendo repercusiones en las diferentes actividades de la sociedad, de la personalidad y del pensamiento. Son cambios más duraderos, y se relacionan con lo que se ha llamado "marcos de pensamiento" de los sujetos, incluyendo otros puntos de vista, habilidades y estrategias. La decisión que nos cabe tomar como educadores, por lo tanto, no se basa en preguntarnos si tenemos que aceptar esta "mente virtual" que incorpora transmutaciones cognitivas - porque esto ya se está produciendo a partir de los nuevos códigos del sistema cultural simbólico- y sucede más allá de nuestras opiniones y actuaciones. Lo que nos deberíamos preguntar como docentes, es de qué manera podemos contribuir en la construcción de estas 
mentes virtuales de nuestros alumnos, muchos de los cuales viven en ambientes impregnados por tecnologías, con el fin de que seamos capaces de brindar la ayuda pedagógica necesaria para que las tecnologías digitales adquieran verdadero sentido educativo. Tal como lo planteaba M. Castells (2001) la sociedad en la que vivimos adquiere progresivamente connotaciones de "sociedad red". Sin embargo, sabemos que estos fenómenos no son lineales ni igualitarios.

A.Clark (2001) explica que los sistemas de pensamiento se expanden tanto por el cerebro biológico como por el sistema de circuitos no biológicos, en el cual se combinan tecnologías externas y habilidades cognitivas internas para la codificación e intercambio de conocimientos. Estos procesos instauran "actualizaciones de la mente", que no solamente incrementan las capacidades cognitivas de los sujetos, sino que también las transforman: aprendemos a pensar de un modo nuevo con la ayuda de las tecnologías.

Sin embargo, esto sucede siempre y cuando existan contextos favorables con respecto a la optimización de las potencialidades de las nuevas tecnologías para el aprendizaje. Si no generamos ambientes culturales propicios para mejorar las propuestas de enseñanza a partir de las variadas posibilidades que ofrecen las tecnologías, la diferenciación en las formas de inclusión social que surgen como consecuencia de su impronta en todas las regiones, será calamitosa. No podemos dejar de tener en cuenta que sólo el $3 \%$ de la población mundial tiene acceso a una computadora, y un porcentaje aún menor utiliza Internet. La inmensa mayoría de las personas que habitan este plantea ignora que existen nuevas tecnologías.

\section{Evoluciones web, ilusiones y problemas de siempre}

Una vez puestos en marcha los servicios de la red Internet, las posibilidades que han surgido en el ámbito educativo han sido altamente promisorias. La Web 1.0., también denominada "fase punto.com" implicó el almacenamiento de una inconmensurable cantidad de contenidos. No obstante, esta primera etapa de Internet se caracterizó principalmente por la transmisión y la recepción de información. La web era una inmensa biblioteca, pero sólo los administradores determinaban el qué, el cuánto y el cómo del acceso a la información. La Enciclopedia Británica virtualizada y Encarta fueron emblemas de esta fase inicial.

La Web 2.0. ha sido llamada la "web social". Los software libres fueron la vedette de esta etapa, en la cual no sólo se brinda información, sino también recursos diversos, los cuales pueden utilizarse en diferentes contextos y se les puede añadir información. Las páginas híbridas con datos propios agregados, las agendas compartidas, las plataformas tecnológicas aplicadas a la educación, las etiquetas que permiten clasificar y ordenar, los blogs, las webquests, el twitter, las wikis, los portafolios y el facebook, entre muchos otros, son productos de esta fase de desarrollo de Internet que actualmente utilizamos en forma masiva. 
La Web 3.0. es también conocida como la "web semántica". Los expertos auguran que este nivel de evolución de las prestaciones de Internet permite que la información sea más comprensible, además de accesible. Las computadoras adquirirán la capacidad de analizar los contenidos, las rutas seguidas y los intercambios efectuados. Toda esta información posibilitará que la máquina elabore perfiles de nuestras características como usuarios para "asesorarnos". Por ejemplo, a partir de los enlaces que hemos realizado y de otros muchos datos que vamos dejando como huellas en el sistema informático, la web semántica nos sugerirá inscribirnos en tal o cual posgrado, según se requiera dominio de algún idioma, sea oneroso para nuestro nivel de ingresos o de bajo costo, incluya espacios curriculares afines a nuestra formación y trayectoria profesional, etc. Los estudiantes serán identificados por esta web sensible, y su prestación se adaptará a la edad de los mismos, a sus conocimientos previos, a sus intereses y experiencias. La computadora, a modo de un "Gran Hermano virtual", registrará todos nuestros recorridos por el mundo de los bits, y nos indicará en el momento preciso que nos hemos equivocado o hemos resuelto mal un problema, mostrándonos también las estrategias más acertadas que utilizamos en otro momento en el que teníamos mayor inspiración cognitiva.

Ante este enorme despliegue de motores de búsqueda semántica para clasificar personas a partir de sus hábitos, huellas ciberespaciales y procederes socio-cognitivos, nos preguntamos si esta mirada panóptica de Internet no generará mayores prejuicios en los ámbitos educativos. En la década del ochenta, T. Robertson y L. Jacobson investigaban el efecto Pygmalion en las escuelas, por el cual los maestros solían estigmatizar a sus alumnos considerándolos buenos o malos según las opiniones recibidas de otros colegas, y enseñando en sintonía con estas pre-concepciones. De este modo, los alumnos etiquetados como inteligentes lograban un rendimiento mayor que aquellos catalogados como lerdos, aún cuando la conformación de los grupos había sido al azar. Estos casos de profecías autocumplidas a partir de prejuicios son muy comunes en nuestras instituciones. Nos inquieta, por lo tanto, el hecho de que se catalogue a través de una red semántica a los estudiantes, que se puedan elaborar perfiles rígidos o estereotipados de los mismos, y que esta clasificación de folksonomías sea considerada como científica e inapelable por los educadores. "La computadora no se equivoca" parece ser un eslogan recurrente en nuestros contextos, y esto está basado en el mito de que la tecnología es superpoderosa y puede resolver los problemas que los humanos no podemos afrontar: "el alumno Juan no pudo resolver en diez minutos el problema de matemática. Lo informó la computadora. El alumno Juan fracasará en matemática".

En ambientes altamente competitivos y tendentes a mantener relaciones despersonalizadas entre docentes y alumnos, estos razonamientos pueden generar un efecto de halo, es decir, de minimización o despreocupación por las tareas de enseñanza en consonancia con la idea de lo inmodificable. 
Estas hipótesis nos llevan a reflexionar acerca de la urgente necesidad de formar a los docentes en los múltiples aspectos que se relacionan con las tecnologías educativas.

\section{La formación de los docentes, un problema complejo}

Existe una marcada tendencia por la incorporación de hardware en los sistemas educativos de todos los países. En las últimas décadas, el incremento en la cantidad de computadoras y en conectividad ha sido sustancial en las instituciones. No obstante, las investigaciones dan cuenta que el uso efectivo de las computadoras para la enseñanza por parte de los docentes es limitado, irregular y parcializado. Un informe de la Organización para la Cooperación y el Desarrollo Económico -OCDE- realizado en el año 2004 reporta que en catorce países relevados, sólo el $20 \%$ de los docentes usaba asiduamente la computadora para sus clases. Sin embargo, la OCDE habla también de una "decepción" respecto de la formación en tecnologías educativas, pues en los países desarrollados estudiados se percibe un predominio de aspectos informáticos en la utilización de TIC en las escuelas secundarias, descuidándose los propósitos de educar para un mundo más justo y solidario.

Resulta por demás evidente que las innovaciones tecnológicas han producido múltiples cambios en la educación. Se han suscitado transformaciones a nivel socio-institucional, político, económico y cultural. Una concepción hegemónica en las propuestas de formación docente relacionadas con las tecnologías educativas, ha sido aquella que ha privilegiado sólo la dimensión técnica. Desde este enfoque, se considera que los cambios que introducen las TIC son siempre beneficiosos e inevitables, en una postura eficientista y reproductivista que asume en forma incuestionable que las tecnologías constituyen el "tren del progreso" al cual hay que subirse para no quedar fuera, en una suerte de moda de pedagógica que no se analiza. Los típicos cursos de informática para el uso operativo de la computadora y para la utilización de los recursos de la web 2.0. (blogs, wikis, plataformas tecnológicas, etc) se encuadran en esta concepción instrumentalista que focaliza la capacitación basada solamente en el manejo del artefacto tecnológico, dejándose de lado otros aspectos.

Si bien la adquisición de conocimientos acerca de las TIC como herramientas es importante, estos contenidos no deben soslayar la reflexión crítica acerca de otras dimensiones, como por ejemplo las decisiones políticas que subyacen a la incorporación de las TIC en la sociedad, sus beneficios, limitaciones e implicancias económicas; las realidades de cada contexto educativo e institucional; la fractura digital que se abre cada vez más entre los bien provistos en tecnologías de la información y los mucho más numerosos desprovistas de las mismas (Ramonet, 2004), el potencial pedagógico y didáctico de las tecnologías, las relaciones entre tecnologías y contenidos disciplinares; la conformación de los proyectos curriculares; las posibilidades y limitaciones de 
los diversos softwares educativos; los problemas que presenta la alfabetización digital; los procesos psicológicos relacionados con el aprendizaje a través de las nuevas tecnologías; las estrategias socio-cognitivas puestas en juego; las características actuales de las culturas juveniles; la incidencia del mercado en la compra-venta-manutención de los productos tecnológicos; etc.

D. Buckingham (2008) observa que una concepción comúnmente aceptada acerca del objetivo de las tecnologías en la escuela ha sido el de "encantar al niño desencantado". No obstante, hay que admitir que actualmente la escuela ya no controla ni la socialización ni la enculturación de los niños y jóvenes, y en muchos casos la cultura del consumo infantil se opone a la que ofrece la educación formal. Podemos constatar que, en términos generales, el uso que se hace de la computadora en la escuela está muy desvinculado de los complejos ambientes tecnológicos saturados de medios en los que se mueven los jóvenes.

Desde estos parámetros, resulta indudable que la escuela debe mejorar el acceso de los estudiantes a la tecnología, utilizando softwares más interesantes y brindando oportunidades para que los diversos medios se empleen de un modo más creativo. Asumir una actitud puramente "celebratoria" con respecto al acceso a las TIC por parte de docentes y alumnos, y planificar propuestas instrumentalistas de formación docente para la utilización de las mismas, conlleva a generar un habitus superficial basado sólo en modas pedagógicas. Una adhesión típica a estos mandatos -sobre todo en instituciones bien equipadas y/o de carácter privado- es lanzarse a una carrera desenfrenada para comprar lo último que salió en el mercado de los aparatos tecnológicos. Otra opción consiste en elegir sólo los dispositivos y softwares "amigables" para los niños y jóvenes, en una suerte de entretenimiento digital que banaliza los contenidos y las estrategias de enseñanza.

La educación en medios tiene que inscribirse en buenos proyectos pedagógicos, rigurosos en sus propósitos y en la calidad de sus contenidos, pero al mismo tiempo motivadores y desafiantes en relación a las enormes posibilidades que ofrecen las tecnologías digitales. L. Vigotsky (1979) explicaba que, una vez que interiorizamos los símbolos podemos, en un plano externo, interactuar con los demás; y en un plano interno, modificar y regular nuestro propio comportamiento. Tomando como referencia esta noción cognitiva de mediación instrumental, consideramos que las tecnologías que se emplean en las propuestas de enseñanza y aprendizaje virtual no deberían ser utilizadas como meros artilugios u objetos neutros, sino como verdaderas estructuras de acción externa que modelan la reconfiguración de los marcos de pensamiento. No obstante, para que esto sea factible se requiere inscribir los proyectos en marcos teórico-ideológicos que valoren el carácter público del conocimiento, y postulen la democratización del acceso a sus diversas formas de circulación. 
Coincidimos con E. Litwin (2005) cuando plantea que las prácticas de la enseñanza no pueden ser analizadas de manera parcializada partir del buen o mal uso que se haga de las tecnologías, pues éstas se inscriben en propuestas didácticas que han sido pensadas para promover espacios de reflexión y comunicación en el aula, a fin de posibilitar la construcción del conocimiento en un entorno de respeto y de ayuda mutua. En estos ciberlugares pensados y diseñados con esmero por especialistas de diversas áreas, los estudiantes pueden interactuar con sus docentes y con sus pares, en un proceso en el que se crean y recrean códigos comunicacionales y sistemas de significados. Estos modos de apropiación situacional del conocimiento denotan una perspectiva moral, pues la enseñanza asume un compromiso con la reflexión crítica, la búsqueda de sentidos y la construcción de respuestas negociadas y compartidas.

Es importante entonces que los proyectos de formación contemplen la adquisición de alfabetizaciones múltiples por parte de los educadores. Esto requiere una sólida capacitación en la utilización de estrategias adecuadas para analizar críticamente textos diversos, que difieren tanto en su función como en sus modos de representación simbólica. Asimismo, resulta imprescindible generar una actitud valorativa con respecto al papel de las tecnologías en la educación, asumiendo compromisos de innovación y mejora en la tarea docente. Para ello será necesario dejar de lado las posturas asépticas en relación a la alfabetización, pretendiendo ser innovadores sólo a partir de la incorporación de habilidades y destrezas descontextualizadas para el procesamiento del lenguaje digital. En la medida en que los docentes adquieran un metalenguaje crítico acerca de las TIC podrán emitir juicios reflexivos acerca de su potencialidad para la enseñanza y el aprendizaje, superando enfoques banales sustentados en la idea de la mera "aplicación" de los recursos tecnológicos.

No obstante, coincidimos con A. Hargreaves (2003) cuando plantea que los docentes deberán aprender a enseñar de modos que a ellos no les fueron enseñados, promoviendo el aprendizaje cognitivo profundo, pues en la sociedad de la hiperinformación los estudiantes, más que información, necesitan adquirir capacidades para saber organizarla y atribuirle significado y sentido. Los docentes también tendrán que asumir el compromiso de formarse de manera permanente, trabajando de modo colegiado en ámbitos de inteligencia colectiva. En las organizaciones económicas se promueve no sólo que los trabajadores sepan sobre algo respondiendo al porqué y al cómo, sino que también se requiere el saber quién sabe algo, y saber quién sabe lo que hay que hacer. Esto implica habilidades sociales de comunicación y de cooperación con personas que cuentan con diversas experticias. En la actualidad, resulta fundamental que los docentes reconozcan y respeten los conocimientos que poseen sus alumnos -como por ejemplo los saberes acerca del manejo operativo de las nuevas tecnologías- para solicitar su colaboración y aprender de ellos. Indudablemente, esto conlleva a modificar actitudes, asumiendo que la 
inteligencia no es singular, fija e individual, sino universal, múltiple, distribuida y compartida.

Desde experiencias compartidas por colegas en diversos niveles de la enseñanza, percibimos que al sistema educativo actual se le adjudican retrasos respecto a las demandas vigentes de la sociedad. No obstante, es preciso reconocer que los esfuerzos que realizan muchas instituciones para incorporar las tecnologías en condiciones altamente desfavorables, son loables. Sin embargo, las formas de participación relacionadas con las mismas dan cuenta de que los propósitos están más orientados a lograr la inclusión de los estudiantes en los cánones de la sociedad de la información, que a modificar realmente las prácticas de enseñanza, asumiendo un compromiso por desarrollar un pensamiento crítico acerca de estas nuevas producciones culturales.

Un aspecto relevante en estos procesos de formación consiste en dilucidar las verdaderas características y posibilidades de las instituciones en las que se desarrollan las nuevas tareas docentes que demanda la enseñanza, en el marco de microcontextos de formación virtual o de propuestas presenciales mediadas por tecnologías. Muchas veces las universidades e institutos superiores programan acciones que no se condicen con su idiosincrasia organizacional, de infraestructura o de previsión presupuestaria. De este modo, los proyectos virtuales o mediados por TIC quedan sujetos a una impronta voluntarista por parte de los docentes y administrativos que asumen la responsabilidad de su puesta en marcha, con el consecuente desgaste físico y emocional que estas tareas demandan. Además, investigaciones realizadas en este campo dan cuenta que las prácticas que predominan en el profesorado relativas a la utilización de TIC son de tipo experimentalista. Se deja de lado la reflexión crítica y la producción teórica, lo cual sólo puede lograrse a partir de la investigación educativa.

Por ello, como decía Célestin Freinet, no debemos ser ni tradicionalistas empedernidos ni ansiosos innovadores, cazadores permanentes de aventuras. Como docentes, tenemos que interesarnos por la utilización de las nuevas tecnologías para enseñar de manera más flexible y comprensiva, adecuando las mismas a nuestros contextos y realidades. Caminaremos con ellas paso a paso, las haremos propias según nuestras necesidades y las de nuestros alumnos; y de este modo podremos escalar montañas.

\section{Referencias Bibliograficas}

- Buckingham D. (2008) Más allá de la tecnología. Aprendizaje infantil en la era de la cultura digital, Buenos Aires, Manantial.

- Castells, M. (2001) Internet y la Sociedad Red, [en línea], Disponible en: www. tecnologiaedu.us.es/bibliovir/pdf/106.pdf

- Clark A. (2001) Natural born cyborgs? [en línea], Disponible en: www.edge.org/3rd_ culture/clark/clark_index.html 
- Claxton G. (1994) Educar mentes curiosas. El reto de la ciencia en la escuela. Madrid: Aprendizaje Visor.

- Coicaud S. (2000) La colaboración institucional en la educación a distancia, en: Litwin E. (comp.) La educación a distancia. Temas de debate en una nueva agenda educativa,(pp. 73-103) Buenos Aires, Amorrortu.

Buenos Aires: Biblos.

- $\quad$ Coicaud S. y Ortega Carrillo J. A. (2007) Escuelas en Red y Ciberescuelas. En: Ortega Carrillo J. A. y Chacón Medina A. (Coord.) Nuevas Tecnologías para la Educación en la Era Digital, (pp. 307-316), Madrid: Ediciones Pirámide Anaya.

- Coll C. y Monereo C. (Eds.) (2008) Psicología de la educación virtual. Madrid: Morata

- $\quad$ Fortunati L. (1997) The Ambiguous Image of the Mobile Phones, [en línea], Disponible en: www.cost269.org/Cost248/0_INTRO.doc

- $\quad$ Freinet C. (1996) La escuela moderna francesa. Una pedagogía moderna de sentido común. Las invariantes pedagógicas. Madrid: Morata.

- Gutiérrez Martín A. (2003) Alfabetización digital. Algo más que ratones y teclas. Barcelona: Gedisa,

- Hargreaves A. (2003) Enseñar en la sociedad del conocimiento. Barcelona: Octaedro

- Litwin E. (comp.) (2005) Tecnologías educativas en tiempos de Internet, Buenos Aires, Amorrortu.

- OECD Organización para la Cooperación y el Desarrollo Económico (2004) Informe: Completing the foundation for lifelong learning, [en línea]. Disponible en: http://www.oecd. org/document/1/0,3343,en_2649_39263238_27443329_1_1_1_1,00.html

- $\quad$ Prensky, M. (2001). On the Horizon. MCB University Press, 9 (5) [en línea]. Disponible en: http://www.marcprensky.com/writing/Prensky\%20-\%20Digital\%20Natives,\%20Digital\%20lmmigrants\%20-\%20Part1.pdf

- $\quad$ Ramonet I. (2004) El nuevo orden Internet, Revista Le Monde Diplomatique, Buenos Aires, Edición Cono Sur, 55, (2).

- Rheingold H. (2004) Multitudes inteligentes. La próxima revolución social. Barcelona: Gedisa.

- Rosenthal N. y Jacobson L. (1980). Pygmalion en la escuela. Expectativas del maestro y desarrollo intelectual del alumno. Madrid: Marova.

- Salomon G. (1992) Las diversas influencias de la tecnología en el desarrollo de la mente, Revista Comunicación, Lenguaje y Educación, Madrid, 13, (13-20).

- San Martín Alonso A. (2009). La escuela enredada. Barcelona: Gedisa.

- Sancho Gil J. (coord.) (2006). Tecnologías para transformar la educación, de Universidad Internacional Andalucía. Madrid: Akal.

- Vigotsky L. (1979) El desarrollo de los procesos psicológicos superiores. Barcelona: Crítica. 